\title{
Online Biscuit Detection System Based On Machine Vision
}

\author{
Wei Xin $^{a}$, Chen Guangfeng ${ }^{b}$, Zhai Linlin ${ }^{c}$, Huang Qingqing ${ }^{d}$ \\ College of Mechanical Engineering, Donghua University, Shanghai 201620, China \\ a'weixin3032503@163.com, ${ }^{b}$ chengf@dhu.edu.cn, 'zhail@msn.com, ${ }^{d}$ huangqq@msn.com
}

Keywords: online detection; machine vision; geometric matching; feature extraction; NI Vision

\begin{abstract}
In order to complete the automated sorting, the manipulator needs the accurate coordinate and angle information of the biscuits. This article design a machine vision based online biscuit detection system. Devise the hardware structure and control logic. Base on geometric matching algorithm, develop the detection software with NI Vision. The software could acquire video to analysis to get the coordinates of biscuits, and update and exchange the data with manipulator control software. The system has been tested to achieve a complete detection rate about $96 \%$.
\end{abstract}

\section{Introduction}

Automation and control products are already widely used in the food packaging machinery industry. However, the level of automation of the control system of packaging equipment in the food packaging machinery industry is not very high now. And for many small food enterprises, it still remains in the stage of artificial packaging, not only leads to the low efficiency, but also affects the food security to some degree.

Several years ago, this auto-recognition function has been used to package products in some foreign food plant, which provides a great convenience for a wide array of products packaging together. Due to the disordered products on production line, the control program needs the scan probe to determine the position of different shape material, then feedback to different manipulator so that the manipulator can put the goods into the plate accurately and correctly according to its position and direction. It achieves rapid and accurate packaging and at the same time, eliminates the manual operation of the visual and physical fatigue and other factors.

This paper proposed an online biscuit detection system to grab the video and extract the positions of biscuits and exchange the information with manipulator control software.

\section{Detection System Design}

System Hardware. As shown in Fig. 1, an industrial camera together with a circular light source is mounted just above the conveyor at an appropriate height. When the conveyor starts, open the industrial camera. The industrial camera continues to pass the collection of the video to a computer. When biscuits appear in the video, detection software detects biscuits' position and angle information and transfers them to the manipulator control software (MCS). After the MCS finish its action, MCS returns a 'true' value to Labview and empties the corresponding data.

Detection Logic Design. Overall design ideas: biscuit video acquisition-> biscuit->recognition ->data refreshment->data communication->data cleanup. The most important of all is the biscuit recognition. The detection and process is showed in Fig.2:

Feature-based Geometric Matching Algorithm. Pattern matching and geometric matching are wildly used for some applications to identify objects and obtain the object information such as the position or angle. These two algorithms have their own advantages and application conditions. When an image has a large amount of information or the outline of objects in the image is very complex, using pattern matching is faster. When the outline of objects in the image is simple and objects' declinations are uncertain, geometric matching algorithm don't need to rotate the template constantly to match, so it would be much faster. 


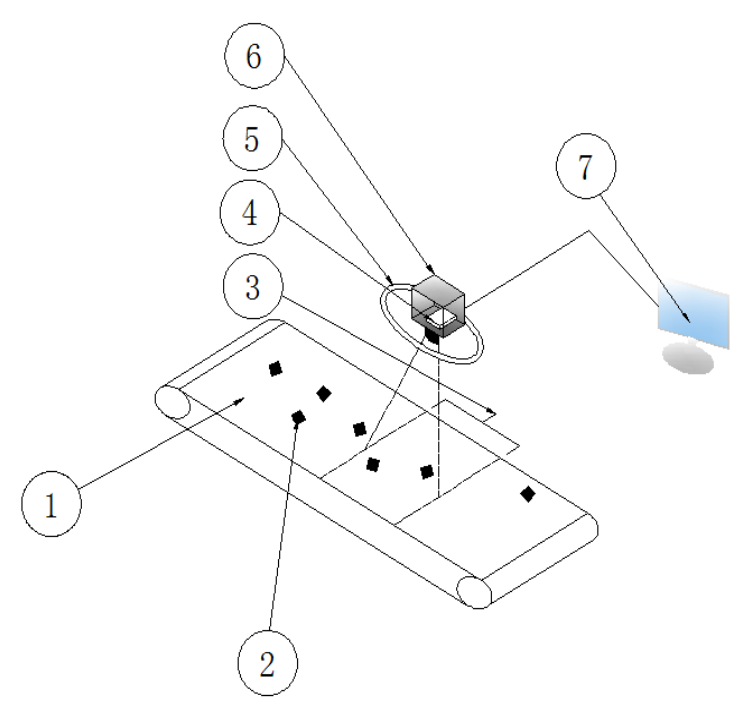

Fig. 1 Hardware of detection system

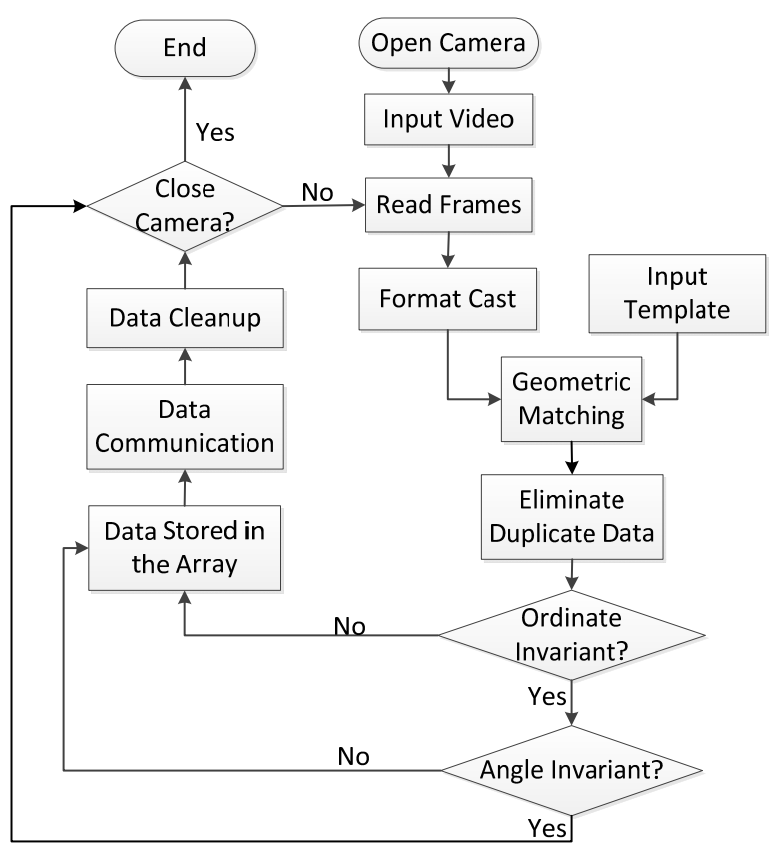

Fig. 2 Detection system control logic

This article is to identify the outline of biscuits, and therefore suitable for using geometric matching algorithm. The algorithm is divided into two stages which called template learning stage and matching stage. In the learning stage, we must first extract curves of a biscuit in the template and these curves compose the typical features such as rectangular features, corners, circular features and linear features. These features are stored in the template image for matching stage. In the matching stage, the algorithm extracts curves in the target image and recomposes the features. If the features extracted from the target image are consistent with those of the template image, we superimpose the template model from the learning step onto a potential match in the inspection image to confirm that the potential match exists or to improve the match. Finally, output the angle, position or other information of the matched object.

Both in learning and matching stage need curve extraction. Fig. 3 is a brief introduction of curve extraction process. Curve extraction is described in detail in Fig. 4. In Fig. 4, (1) are scan lines, (2) is a row step, (3) is a column step, (4) is a curve seed, (5)are curves. To increase the speed of the curve extraction process, the algorithm visits only a limited number of pixels on the scan lines in the image to determine if the pixel is a valid seed point.

Enhanced Algorithms. Biscuits' appearances in the range of positions of the camera lens are uncertain and therefore the Region of Interests (ROI) can't be accurately divided. If the ROI is too large, some biscuits would appear twice in the region, resulting in duplicate detection. When ROI is too small, some biscuits might no longer appear in this area, resulting in undetected case. To resolve this problem, the ROI can be set large enough so that all biscuits can appear in this area at least one time, and then exclude the duplicated biscuits. In this case, each of the biscuits needs a 'tag' in order to let the program know which has been detected. This 'tag' should be inherent of each biscuit.

Labview vision module operates on the pixels of the image with very high accuracy. So the abscissa of each biscuit can be its inherent 'tag'. The middle part of the range of the lens in the video is lighter, so the brightness changes of the images will affect the accuracy of the coordinate values of detection, but its impact is limited. Setting a tolerance range can overcome this disadvantage (the tolerance range is set to 10 pixels). The only flaw of this approach is that if consecutive two biscuits' horizontal coordinate spacing is less than 10 pixels, it'll cause undetected case. When we tested again, this condition really appeared and it caused the missing rate of 1/15. 


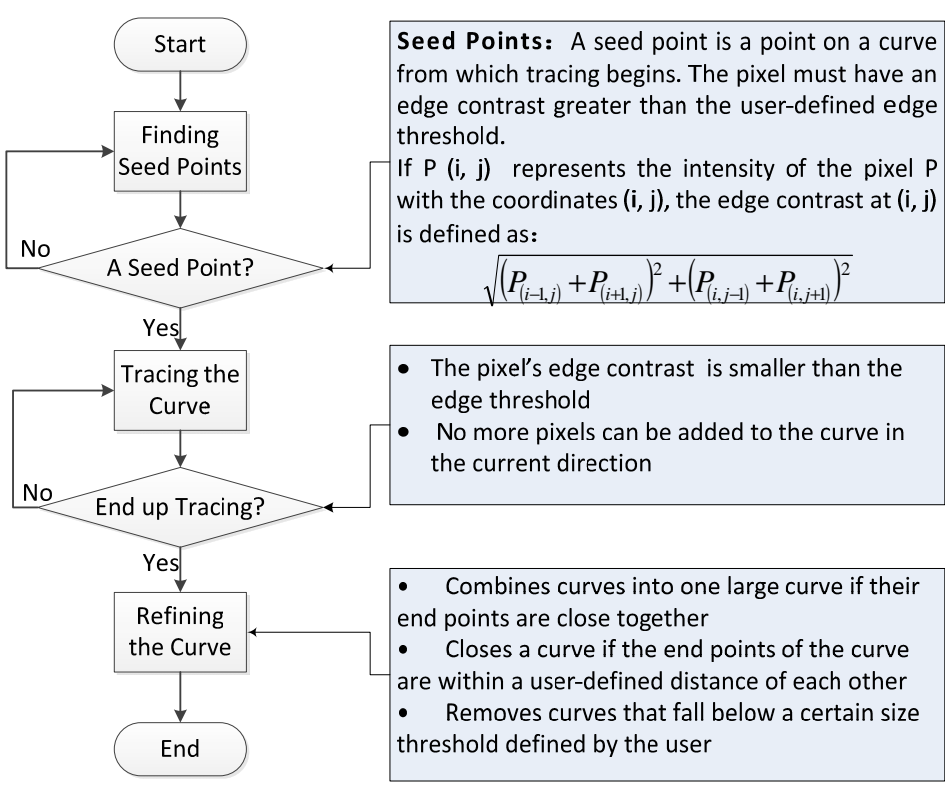

Fig. 3 Curve extraction process

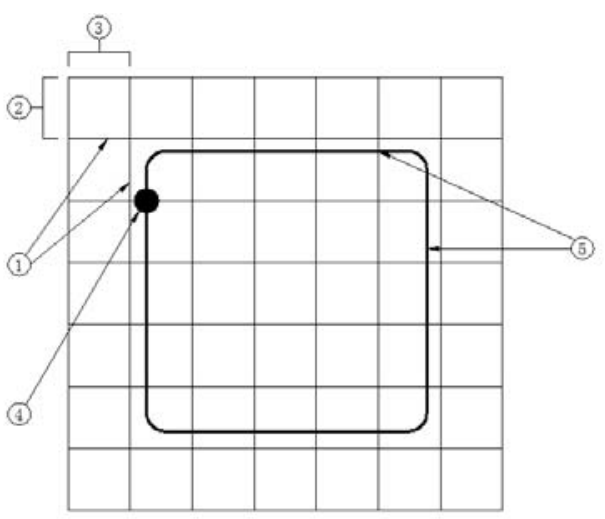

Fig. 4 Curve extraction

In fact, the biscuit has another inherent 'tag'-angle. When two biscuits have the same abscissa, we also need to see if the angle deviation is within the tolerant range. Once angle variation between the two biscuits is also within this range, those two are supposed to be same biscuit, and discard this group of data. If the angle variation is outside this range, they are supposed to be two biscuits, and this group of data is still valid. By this mean, the system will greatly reduce the undetected cases.

Data Refreshment. There is a distance between the manipulator's working area and the industrial camera's detection position. So the biscuits' coordinate data need to be refreshed constantly after finishing detection. Considering the conveyor's speed, as well as the time interval between the frames is known, and then we can calculate the ordinate changing value. This value will be added to the biscuits' original ordinate after the program puts the new coordinate data into an array in order to achieve data refreshment.

Data Communication. There are several communication methods in Labview. This system adopt TCP protocol to realize network communication between Labview and manipulator control program. The manipulator is controlled by the MCS, so Labview only needs to transmit the biscuits' coordinates and angles to MCS and receives the bit information in which the data needs to be clean up. When Labview is used as a server, the transmission process is shown as follows:

Start->Open TCP Connection-> Type Cast-> TCP Write Data Size->TCP Write Data->TCP Close Connection->End.

Data Cleanup. The program will check up whether the cleanup by bit button is valid after acquiring the coordinate and angle data of the biscuits. This button is a Boolean control. When manipulator control software returns a 'True' value, this button is valid and then Labview clears the corresponding data in the array. If the button is invalid, nothing will be done to the array.

\section{Testing and Discussing}

System Test. The proposed system was tested to verify its ability. At first, we didn't use the enhanced algorithm and calculated the ordinate transformation value of the biscuit between two frames based on the speed of the conveyor. We determined the ROI according to the value to ensure each biscuit appears in the ROI only once. However, the performance wasn't ideal for there had been a series of biscuits undetected cases. Fig. 5 was one of the cases that biscuits were undetected.

The condition was improved while detection with the enhanced algorithm, as it was shown in Fig. 6. Through comparing between Fig. 5 and Fig. 6, the enhanced algorithm solves the problem of un-detection. The geometric matching algorithm can find a template match in an inspection image even when the match is partially occluded because of overlapping parts or the part under inspection not fully being within the boundary of the image. The maximum occlusion rate is upto $50 \%$. Before corrected, due to the limitation of ROI, the occlusion rate can't be too large. Large will cause the 
duplicate detection and small will cause undetected cases as shown in Fig. 5. The improved algorithm doesn't need to consider the impact of ROI, so we can increase the value of the occlusion rate. After playing the video in 20 loops, statistic show that undetected rate and repetition rate were $1 / 50$ and $13 / 600$ respectively.

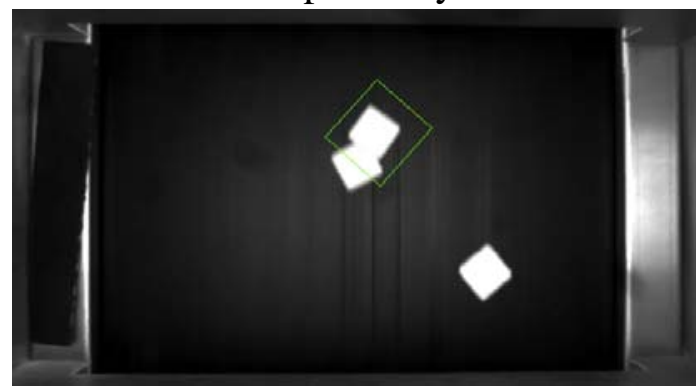

Fig. 5 Undetected case

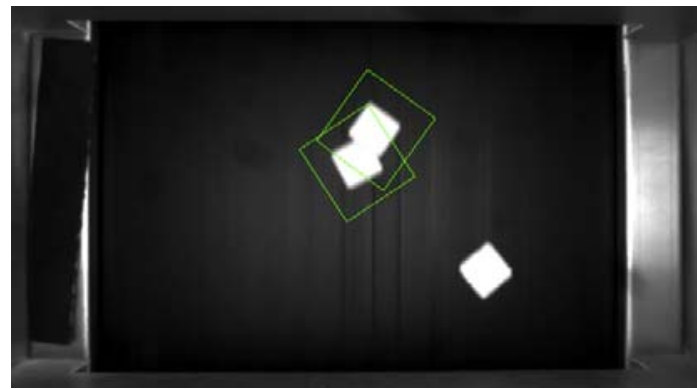

Fig. 6 Detection after improving the algorithm

\section{Conclusion}

This paper proposed a machine vision based online biscuits detection system. Devise the hardware structure and control process. Base on geometric matching algorithm, develop the detection software with NI Vision. The software could acquire biscuit video to process the video to get the coordinates of biscuits, and update and exchange the data with manipulator control software. The test results show that the system has a high reliability for biscuits detection in conveyor.

\section{Acknowledgements}

This work was supported by Fundamental Research Funds for the Central Universities (12D10309).

\section{References}

[1]Jeffrey Travis, JimKring. Labview for Everyone. Beijing: Publishing House of Electronics Industry, 2011, third edition

[2]Mark S. Nixon, Alberto S. Aguado. Feature Extraction and Image Processing. Beijing: Publishing House of Electronics Industry, 2010, second edition

[3]He Xiaowu, Du Jian. Status Quo and Development Trend of Use of Automation Control Products in Food Packaging Machinery Industry. The Beverage Industry, 2011,(5): 41-43

[4]Gao Bintang, Liu Jianhua. Dynamic Data Exchange Between Labview and VC Program. Industrial Control Computer, 2009, (12):26-28

[5]Liang Lvjie. Research on the Application of Mechanical Automation in Food Packaging. Mechanical Research \& Application,2011, (02):4-6

[6]Zhu Zuofu, Xu Chao, Ge Hongmei. Applications of Machine Vision in Packaging. Packaging Engineering, 2010,(3):124-127 